\title{
On the Characteristics of Migrant Children's Class Network in Beijing
}

\author{
Yan Ming \\ Department of Sociology and Social Work, Capital Normal University, Haidian, Beijing, China \\ mingyan@cnu.edu.com
}

\begin{abstract}
This paper analyzes 2,054 migrant and 700 local children in 11 schools of Beijing to explore the basic features of social network, structure of class network, and the roles and determinants of migrant children in the class. The main conclusions are: the schools recruiting both migrant and local children overall promote exchanges between the two groups, but on the individual, migrant children face the higher risk of isolated in mixed classes. To some extent, migrant and local children's integration seems selective. Family relationship, family control and academic performance mainly influence the roles of migrant children in their class.
\end{abstract}

Keywords - migrant children, class networks

\section{北京市流动儿童班级社会网络特征分析 \\ 明艳}

首都师范大学政法学院社会学与社会工作系, 海淀, 北京, 中国

\begin{abstract}
摘 要 本文使用对北京市 11 所学校、94 个班级、 2054 名流动儿童及 700 名对照组京籍儿童的调查数据, 探讨了流动儿童社会 网络的基本特征、流动儿童在班级网络中的角色及影响因素, 并对班级网络结构做了分析。主要结论有: 混招学校总体上促进了京籍 与流动儿童之间交往, 但就个体而言, 流动儿童在混合班级网络中被隔离的风险更高。京籍儿童与流动儿童两个异质群体表现出相当 程度的的交往融合, 但目前看来, 这仍是一种有选择的融合。流动儿童是否是班级领袖的影响因素中, 家庭关系的亲密水平影响力最 大, 而在流动儿童是否成为班级边缘成员的分析中, 成绩越差、家庭关系越冷漠, 家庭控制越专制, 流动儿童有越高的可能性成为班 级中不被认可、被边缘化的成员。
\end{abstract}

关键词 流动儿童, 班级网络

在中国城市化的进程中, 随着人口流动的规模越来越 大, 平均居留时间越来越长, 流动家庭化 (主要是核心家 庭, 即父母与自己的未成年子女）的趋势也逐渐呈现且日 益明显。流动儿童的社会网络是社会融合水平的重要体现。 网络特征与结构可以反映流动儿童拥有或缺失的支持性资 源、反映其在生活情境中角色与位置, 而这将影响他们在 现住地的生存状态、心理情绪、以至未来发展路径。分析 流动儿童社会网络, 既是对这一群体本身的关注, 更宏观、 长远地看, 也是对城市良性和谐发展的关注。

\section{1. 文献回顾}

关于社会网络的研究众多, 它关注的是人们之间的互 动与联系。项逐（2000）认为这些流动人口的工作、生活、 娱乐都植根于他们的社会网络, “越边境的社区” 是他们 城市生活的主要场所。赵延东 (2002) 在实证研究中也提
出在农民工经济地位获得过程中, 社会网络所扮演的角色 是极其重要的。王毅杰 (2005) 认为 “规模小、紧密度高、 趋同性强、异质性低” 是流动农民工社会网络的主要特点。 李汉林 (2002) 认为农民工群体以初级群体为基础的社会网 络具有 “强关系” 的特点, 指出同质群体成员是构成农民 工之间强关系纽带的基础。李培林 (1996) 发现, “亲缘关 系网络” 作用贯穿于流动人口生活和交往的整个过程, 制 约了就业方式、生活交往方式, 决定了流动民工的内部分 层和社会分层中的经济社会地位, 进而影响流动民工的生 活满意度等。这种 “亲缘” “地缘” “情缘” 的社会网络 在流动人口融入社会生活初期起到了积极作用, 在一定程 度上防止其沦为城市化失败者, 在另一方面却强化了农民 工生存的亚社会生态环境, 保护了农民工身上所具有的传 统观念和小农意识, 阻碍着其对城市的认同与归属。

\section{从已有的研究来看, 研究对象上, 社会网络的研究基}


本都是针对流动人口, 专门针对流动儿童的研究比较少见。 研究数据和方法上, 定性研究多于定量研究, 定量研究中, 常见的统计学分析比较多, 而运用社群图分析方法的较少。

不同于成人社会网络的立体、多层次, 6-14 岁流动儿 童的社会网络资源主要包括学校资源, 同龄群体, 尤其是 同班同学。基于此, 本文将主要关注流动儿童的班级社会 网络——这种通过同学互动而形成的相对稳定的体系。这 可以将流动儿童保留在其日常生活的情境之中, 继而比较 完整地了解个体之间的关系本质。

\section{2. 研究设计}

\section{1 研究数据}

本文使用的数据包括两类: 一是全部数据, 课题组 2010 年在北京采取重点抽样和多阶段与规模成比例抽样 (PPS) 调查相结合的方法, 在西城、朝阳、丰台、通州、 昌平 5 区县 11 所学校开展调查, 包括 4 所纯流动儿童校、 5 所流动儿童居多的混招学校、2 所京籍儿童居多的混招学 校。共调查 94 个三年级以上的班级, 包括 2054 名 6-14 岁 流动儿童及 700 名对照组京籍儿童, 以他们为研究对象, 分析流动儿童社会网络的基本特征。

二是班级数据, 通过被调查儿童对 “你最好的朋友是 谁” 问题的回答, 本文整理出了样本中的 11 个班级共计 494 名同学, 分析流动儿童在班级网络中的角色与位置。

\section{2 研究方法与内容}

运用定量研究和社群图分析方法, 本文对流动儿童社 会网络、尤其是班级网络的特征与结构展开分析, 包括 3 个方面: 流动儿童社会网络的基本特征; 流动儿童在班级 网络中的角色及影响因素; 班级网络结构分析。

\section{3. 流动儿童社会网络的基本特征}

3.1 流动儿童班级网络中亲密同伴的平均规模不少, 但是 没有被指认为朋友的人比例比京籍儿童高

社会网络规模（ size of social networks）指构成 一个社会网的成员数目。一个人的社会网规模越大, 他所 拥有的社会资源越丰富 (林南, 1982)。社会网规模也是测 量个体社会资源拥有程度的一个重要指标。

而对于流动儿童来说, 同伴关系是他们建构社会网络 的主要影响因素。方差分析发现打工子弟学校和公立学校 的同学在亲密同伴的交往规模上没有显著的差异。大部分 的同学都会被指入和指出朋友 (指入意为被别人选择做最 好朋友, 指出意为指认别人为朋友)。被 3-10 名同学指认
为最好朋友的同学占到总数的 $66.5 \%$, 能够指认出自己的 5 名最好朋友的同学也占了 $79.6 \%$, 这说明大部分同学都有 自己的亲密同伴, 且规模在 3-10 名之间。

但是在指入方面, 流动儿童中有 $5.8 \%$ 的人在班上没有 一个同学认为 “他” 是朋友, 而相应的比例, 京籍儿童只 有 $2 \%$ 。这显示出某些流动个体在一定程度上的 “被隔离” 。

\section{2 流动儿童的情感支持网络比较缺失}

社会网络的一个重要作用就是情感支持, 本文对流动 儿童的情感支持网络进行了特别的关注。流动儿童在有心 里有话要对别人说的时候, 选择最多的是同学 (28.1\%) 和 父母 (20.9\%), 可以看出, 对于流动儿童来说, 学校、家 庭仍然是他们最重要的情感成长环境。值得关注的是, 有 相当多的孩子（14.9\%）选择了 “谁也不说”, 我们推测, 除了这些孩子过于内向不愿说之外, 受到老师有意无意的 忽视、家长因为工作忙而造成的无意忽视等而无处可说可 能是更重要的原因。

这一问题同样在 “现在你生活中感觉最苦恼的问题是 什么” 中有所体现。流动儿童中选择 “父母没时间照顾” 的比例 (6.5\%) 要比京籍儿童选择此项的比例 (2.5\%) 高 出一倍多。流动人口来到北京需要为生活打拼, 而没有很 多的时间和精力放在孩子的身上, 这在一定程度上影响了 流动儿童的心理成长, 可能会造成一些自卑感, 从而不愿 与别人交流, 才有了 “心里话” 从不和任何人讲起。

关于 “有心里话对谁说” , 本文还对流动儿童和京籍 儿童进行了比较, 经过卡方检验, 本文发现他们有显著差 异。其中, 流动儿童在有心里话时跟父母和祖父母说的比 例比京籍儿童低 $8.7 \%$, “谁也不说” 的比例又高 3.5\%。说 明相对于京籍儿童而言, 家庭成员的支持也确实不够。

3.3 给予学习支持的网络成员京籍儿童更多的是父母, 流 动儿童更多的是同学

学习生活是所有儿童最主要参与的活动之一。在流动 儿童的社会网络中, 也有着对于其学习起到支持作用的因 素和成分。本文调查在有不会的作业时学生会问谁, 只有 $5 \%$ (京籍儿童）、 $4.7 \%$ (流动儿童）的调查对象表示 “谁 也不问”, 这说明大部分孩子都有在学习上求助的对象。

流动儿童和京籍儿童的求助对象他们有显著差异。流 动儿童求助的第一对象是同学 (34.6\%), 比京籍儿童高 $3.1 \%$, 而京籍儿童求助的第一对象是父母 (35\%), 比流动 儿童高 $5.1 \%$ 。这说明, 流动儿童的家长可能因为文化程度 低或者因为工作时间太长, 没有过多过问流动儿童的学习。 对流动儿童而言, 在学习方面, 能对他们起到较多帮助的 是身边同学。 
表 1 流动儿童的学习支持网络 ${ }^{1}$

\begin{tabular}{|c|c|c|c|c|c|c|c|c|}
\hline & \multicolumn{7}{|c|}{ 你有听不懂或不会做的作业时, 你第一个会问谁 } & \multirow[t]{2}{*}{ 合计 } \\
\hline & 父母 & $\begin{array}{l}\text { 爷爷奶奶/ } \\
\text { 姥姥姥爷 }\end{array}$ & \begin{tabular}{|l|} 
兄弟 \\
姐妹 \\
\end{tabular} & 老师 & 同学 & 邻居 & $\begin{array}{l}\text { 谁也 } \\
\text { 不问 }\end{array}$ & \\
\hline \multirow{2}{*}{$\begin{array}{l}\text { 京籍 } \\
\text { 儿童 }\end{array}$} & 232 & 12 & 42 & 134 & 209 & 1 & 33 & 663 \\
\hline & $35.0 \%$ & $1.8 \%$ & $6.3 \%$ & $20.2 \%$ & $31.5 \%$ & $0.2 \%$ & $5.0 \%$ & $100.0 \%$ \\
\hline \multirow{2}{*}{$\begin{array}{l}\text { 流动 } \\
\text { 儿童 }\end{array}$} & 603 & 17 & 153 & 427 & 699 & 27 & 94 & 2,020 \\
\hline & $29.9 \%$ & $0.8 \%$ & $7.6 \%$ & $21.1 \%$ & $34.6 \%$ & $1.3 \%$ & $4.7 \%$ & $100.0 \%$ \\
\hline
\end{tabular}

$\mathrm{P}=0.006$

3.4 由于先验标签化, 混合班流动儿童自认的好朋友户籍 选择性很强, 学校性质对于打破儿童交往之间的户籍 界限有益

本文对流动儿童的信息与其最好朋友的信息进行了 配对分析，以此来发现其二者之间的趋同性。

分析表明, 流动儿童最好的朋友中 $86.4 \%$ 为流动儿童, 而仅有 $13.6 \%$ 为京籍儿童。与流动儿童不同的是, 京籍儿 童在选择朋友时就没有明显的户籍选择性, 在其最好的朋 友中, 京籍儿童与流动儿童比例分别为 $52.8 \%$ 和 $47.2 \%$ 。这 一方面可能是由于流动儿童之间有共同的背景和语言, 也 同样有来到城市的不适应感受, 还有对家乡思念等感情上 的共鸣, 这些只有同为流动儿童的同学才可以体会了解; 另一方面, 这在某种程度上反映出流动儿童的一种心理定 势或先验标签化, 可能由于流动儿童为相对弱势的群体, 加上有些歧视性的态度或者制度, 使得某些流动儿童会产 生自卑感, 因此导致其在与同学的交往中会首选与同为流 动儿童做朋友。

考虑到不同类型的学校中流动儿童与京籍儿童的比 例不同, 本文又分别对流动儿童居多和京籍儿童居多的两 种混招学校做了分析, 结果显示, 在流动儿童居多的混招 学校, 流动儿童最好朋友中 $90.2 \%$ 为流动儿童, 在京籍儿 童居多的混招学校, 流动儿童最好朋友中 $66.7 \%$ 为流动儿 童。在京籍儿童居多的学校, 流动儿童有更多的京籍儿童 好友, 主要原因是他们有机会更多地接触到京籍儿童, 这 也在客观上反映出在儿童阶段, 只要有共同学习、互相接 触的机会, 流动儿童与京籍儿童的交往是可以在学校这一 特定情境中得到促进。

\section{4. 学生在班级网络中的地位类型及影响因素}

根据上述的 “亲密同伴的网络规模” 中指入数（被指 认为朋友) 和指出数 (指出别人为朋友, 要求指出 5 人以内) 的数据分析, 同时依照社会网络结构中每个孩子在班级中 的作用, 本文把上述选取的 12 个班级的学生按照 “地位类

\footnotetext{
${ }^{1}$ 本文表格数据来源均为 “北京市流动儿童状况调查” 课题组调查数据
}

型” 进行了分类，共分为五类，分别为:

领袖型 $(6.5 \%)$ : 指入数 - 指出数 $>=5$, 每班约 $1-3$ 人

二等领袖型 $(13.8 \%)$ : $2=<$ 指入数 - 指出数 $<5$, 每班约 3-6 人 边缘型 $(15.8 \%)$ : 指入数 - 指出数 $=<-3$, 每班约 3-7人 绝对边缘型 $(3 \%)$ : 指入数 $=0$ 或指出数 $<5$, 每班约 $1-3$ 人 普通型 $(60.9 \%)$ ：除上述四类的其他同学

4.1 流动儿童比例越低的学校, 流动儿童成为边缘型 (绝 对边缘型) 的比例越高, 成为领袖型 (二等领袖型) 的比例越低

整体上, 流动儿童各种类型学生的比例与京籍儿童差 别不大, 但是不同的学校中, 情况差别较大, 反映出流动 儿童不同的班级处遇。

在打工子弟学校中, 流动儿童中没有绝对边缘型, 边 缘型比例较低 (16\%); 而在流动儿童居多的混招学校中, 绝对边缘型和边缘型学生占 $17 \%$, 在京籍儿童居多的混招 学校中, 绝对边缘型和边缘型学生占到了 $22 \%$ 。

同时, 在打工子弟学校、流动儿童居多的混招学校中, 流动儿童成为领袖的比例为 $7.4 \%$, 而在京籍儿童居多的混 招学校中, 这个比例则只有 $4.3 \%$ 。

结合上文的分析, 本文可以得出结论, 混招学校一 方面在一定程度上促进流动与京籍儿童之间的交流与互 动, 但是, 有一部分流动儿童容易被边缘化。根据个案访 谈, 发现容易被边缘化的儿童大多学习成绩不好, 且无论 家庭经济好坏, 家庭关系都比较疏远冷漠。

4.2 无论流动儿童还是京籍儿童, 学习好、学习态度认真 的同学易成为领袖

在学习成绩方面, 本文发现, 领袖型同学比其他类型 的同学在语数外成绩上都表现较好, 平均分上上也整体上 高 7 分。学习成绩是测量学生学习效果的重要指标, 也证 明着学生的学习能力水平。一般而言, 成绩好的同学会受 到老师、同学、家长的更多关注和鼓励, 也会比较自信, 而这对他们成为领袖型的同学无疑是有利的。

表 2 不同类型学生的学习成绩差异

\begin{tabular}{|c|c|c|c|}
\hline & 平均语文成绩 & 平均数学成绩 & 平均外语成绩 \\
\hline 领袖型 & 86.8150 & 82.8300 & 82.5707 \\
\hline 非领袖型 & 79.8539 & 75.1750 & 75.1750 \\
\hline$P$ & 0.000 & 0.001 & 0.044 \\
\hline
\end{tabular}

除了学习成绩之外, 学习态度也是学业表现的重要方 面。本文对发现能否按时完成作业与学生们的类型有显著 的相关 (卡方检验 $\mathrm{p}$ 值 0.001 ) 。从普通型到领袖型同学 $80 \%$ 左右都能按时完成作业; 而边缘型的同学能按时完成作 业的只有 $65.4 \%$, 绝对边缘型的同学更是下降到了 $46.7 \%$ 。 


\section{3 积极的学校环境会使流动儿童倾向于成为领袖型}

积极的学校环境会对学生类型的形成起到促进作用。 本文以 “得到较多表扬或鼓励” 作为积极环境的标志, 分 析发现, 是否得到更多表扬鼓励和流动儿童的类型是显著 相关的。领袖型学生有 $78.1 \%$ 都能得到较多表扬, 而绝对 边缘型的学生就会比较少 (53.3\%)。值得关注的是, 绝对 边缘型的学生相对于二等领袖型 (57. 4\%) 、普通型学生 (59. $2 \%$ ) 来说, 差别并不是太多, 反而是边缘型的学生得 到的鼓励和表扬最少 (42\%)。这可能是老师在自然关注了 领袖型学生、专门关注了绝对边缘型学生之外, 较少注意 这些不是特别边缘但又不那么能融入集体的学生身上。

\section{4 流动儿童的同学交往认知与其类型有很大关系}

个人对自己社会交往的认知对于他在社会网络中的 地位、所发挥的作用等都有着重要的影响。本文主要通过 三个问题来反映不同类型的学生对自我社会交往的认知和 感受, 包括 “你认为自己容易和同学相处吗”、“大多数 同学对你怎么样”、“你觉得有人燋不起你吗”。

交叉分析表显示, 领袖型和二等领袖的学生普遍认为 和同学相处很容易（分别是 $62.5 \%, 63.2 \%$ ）, 而绝对边缘 型的学生则只有 $20 \%$ 的同学认为很容易。这说明在同学相 处时感受以及自己的认知可能会影响到学生在交往时的策 略和质量, 进而影响到其社会网络中的地位。

对同学是否友好的认知和感受与学生在社会网络中 发展出的类型也有显著关系。领袖型同学有 $62.5 \%$, 二等 领袖同学有 $63.2 \%$ 认为别的同学对自己 “很友好” , 而边 缘型同学只有 $34.6 \%$, 绝对边缘的学生更是下降到 $13.3 \%$ 。 这也说明对于其他人与自我关系的认知明显与交往有关。

经过卡方检验发现, 对别人是否看不起自己的认知和 感受与学生在社会网络中发展出的类型也有显著差异 ( $p=0.006)$ 。领袖型的同学不会普遍觉得别人看不起自 己, 只有 $13.6 \%$ 的同学做这样的回答, 而绝对边缘型的同 学有 $80 \%$ 认为别人看不起自己。

\section{5. 流动儿童班级网的结构分析：一个案例}

本文选取一个班级整体网结构的案例, 来分析京班级 成员的互动模式与发挥的作用, 发现:

在这个 29 人的京籍儿童为主的案例班级中 (京籍 21 , 流动 8), 呈现出两集团型的结构: 分别有 2 个京籍儿童 领袖和 1 个流动儿童领袖, 主导着 $A 、 B$ 两个相对独立的小 团体, 但是小团体内部并不是完全按户籍分割。事实上, 同学交往既存在户籍界限, 又有消融的可能性。同时也充 分说明, 在班级的被认可程度, 并非完全由户籍因素决定。 在 $\mathrm{A}$ 集团中, 主要支持性力量都集中在领袖和二级领袖身 上, 京籍领袖 $\mathrm{a}$ 在学习与情感上都得到成员信任和认同。 而 B 集团的支持性力量则比较分散, 没有完全集中在领袖
身上, 而且其领袖 $\mathrm{a} 2$ (流动儿童) 更偏向于提供情感支持型。

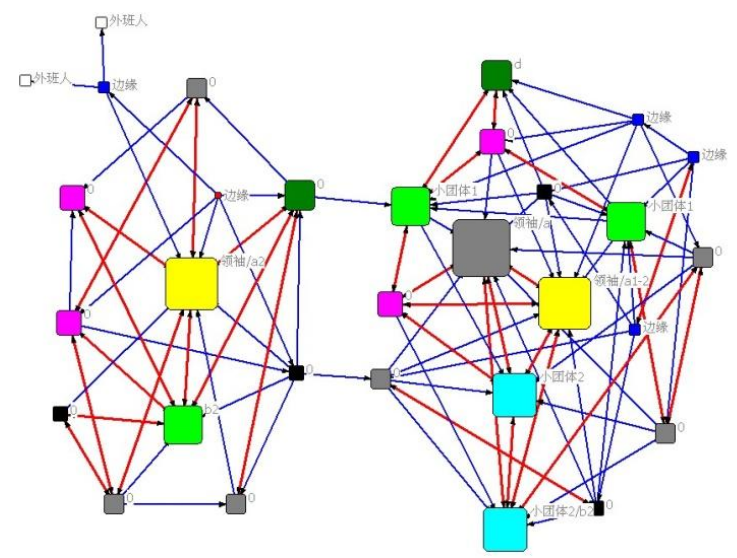

图 1 京籍儿童为主的班级

\section{6. 讨论}

经过以上分析可以看出:

流动儿童自认和他认的亲密同伴的平均规模不比京 籍儿童少。但由于先验的户口标签化, 无论是流动儿童还 是京籍儿童, 自认的亲密伙伴均有一定的户籍选择性, 流 动儿童的选择性更强。

客观地, 混招学校总体上促进了京籍与流动儿童之间 交往, 尤其是在公立的、教学秩序、教学质量较高的混招 学校。但就个体而言, 流动儿童在混合班级网络中被隔离 的风险更高。应该注意这种双向的影响。

流动儿童的经济求助对象、京籍儿童的学业求助对象 分别出现了分化, 这意味着京籍儿童与流动儿童两个异质 群体表现出相当程度的的交往融合, 但目前看来, 这仍是 一种有选择的融合。

流动儿童在班级网络中会分化为领袖型 (6.5\%)、二等 领袖型 $(13.8 \%)$ 、边缘型 (15.8\%)、绝对边缘型 (3\%) 和普通 型 $(60.9 \%)$ 。影响因素包括学校类型、学校环境、学业表现 和同学交往认知。

\section{参考文献}

[1] Biao Xiang, A Community crossing boundary-life history of Zhejiang Village in Beijing, BJ: SDX Joint Publishing Company, 2000.

[2] Yandong Zhao, Fenyu Wang, "Migrants' economic status and its determinants", Population Science of China, no 4, pp8-15, 2002

[3] Yijie Wang, Yunge Ni, "A discussion on social identity of the migrants", Academic Journal of Suzhou University, no 2, pp8-15, 2005.

[4] Hanlin Li, "Guanxi and virtual community --A Perspective of migrant workers", in Migrant workers: Chinese migrant workers' social and economic status, BJ: Social Sciences Academic Press, 2003.

[5] Peilin Li, "The migrant worker's social network and social status", Sociological Research, no4, pp42-52, 1996. 